\title{
LEPIDOPTERA TORTRICIDAE FROM CALABRIA (SOUTHERN ITALY)
}

\author{
(*) Department of Agriculture, Environmental and Food Sciences, University of Molise, Campobasso, Italy \\ (**) Consiglio per la ricerca in agricoltura e l'analisi dell'economia agraria, Research Centre for Forestry and Wood, \\ Rende, Italy \\ $\left({ }^{\circ}\right)$ Corresponding author: trema@unimol.it
}

Trematerra P., Goglia L., Scalercio S., Colacci M. - Lepidoptera Tortricidae from Calabria (southern Italy).

A list of 181 species of Lepidoptera Tortricidae recorded during several expeditions realized in Calabria (southern Italy) is reported. Fifty-nine species are mentioned for the first time in Calabria. Of particular interest are 23 species that have as a southern limit of their distribution area the Calabria territory.

KEY WoRDS: Lepidoptera Tortricidae, fauna, Calabria, southern Italy.

Over time, the Lepidoptera Tortricidae from the Calabria territory (southern Italy) have received sporadic attention by both local and foreign entomologists. The only specific works refer to some catches taken on the southern slope of the Pollino Massif by Trematerra (1990 and 1991a) and in a chestnut area of the Sila Mountains by TREMATERRA et al. (1994).

In the present paper, we reported a tortricid list as updated and complete as possible for Calabria. For this purpose, in addition to our specimens collected from 2013 to 2018, we included in this list both the species found by P. Trematerra in the course of previous entomological researches in that region and the species reported in literature; these last species were collected in an unreported number of samples by light and pheromone traps (TREMATERRA, 1991a,b and 2003; Trematerra et al., 1994 and 1997). In the list, the species from literature are marked with an asterisk.

Our specimens were caught mainly by attraction to light at night-time, mostly using UV-LED light traps (INFUsINO et $a l ., 2017)$, but also by butterfly net during day-time. The material was identified morphologically; genitalia were prepared using standard methods, the abdomen was macerated in $10 \% \mathrm{KOH}$ and dissected under a stereoscopic microscope, the genitalia were separated and mounted in euparal on a glass slide. All specimens and slides were deposited in the Trematerra Collection (at the University of Molise, Campobasso, Italy). From 2013 to 2018 the localities visited were especially in the northern and central part of the Calabrian territory, as Pollino Massif, Sila Mountains and the bordering areas from sea level up to $1500 \mathrm{~m}$ of altitude (Figures I and II; Table 1).

In the compilation of this inventory we followed the systematic arrangement adopted by RAzOWSKI (2002 and 2003 ) with some modifications suggested by BROwN (2005) and by GILLIGAN et al. (2014). Distribution of the species was largely taken from RAZOWSKI (1970), TREMATERRA (2003) and AARVIK (2013), with new data from Trematerra's personal catalogue (unpubl. data). Chorotype of the species was taken from Vigna TAgLianti et al. (1992) and PARENZAN (1994).

\section{LIST OF TAXA}

\section{FAMILY TORTRICIDAE}

\section{SUBFAMILY TORTRICINAE}

Tribe Tortricini

Tortrix viridana Linnaeus, 1758

Material examined: 2 males, Rende (Cosenza), 205 m, 14.V.2014, leg. Scalercio.

Chorotype: W-Palaearctic.

Aleimma loeflingianum (Linnaeus, 1758)

Material examined: 2 males, Montalto Uffugo (Cosenza), 230 m, 24.V.2014, leg. Scalercio; 1 male, San Fili (Cosenza), 831 m, 25.V.2017, leg. Colacci, Goglia and Scalercio.

Chorotype: W-Palaearctic.

Acleris abietana (Hübner, [1819-1822])

Material examined: 1 male, Vivaio Sbanditi (Cosenza), 1350 m, 11.V.2014, leg. Scalercio and Infusino; 1 female, S.S. Bruno (Vibo Valentia), 917 m, 6.IV.2016, leg. Scalercio and Infusino.

Chorotype: Sibiric-European.

Acleris sparsana ([Denis and Schiffermüller], 1775)

Material examined: 1 female, Vivaio Sbanditi (Cosenza), 1350 m, 27.X.2014, leg. Scalercio; 1 female, Lungro (Cosenza), 1270 m, 26.X.2016, leg. Scalercio and Infusino.

Chorotype: European.

Acleris rhombana ([Denis and Schiffermüller], 1775)

Material examined: 1 male, Vivaio Sbanditi (Cosenza), 1350 m, 13.X.2014, leg. Scalercio; 1 male, Rende (Cosenza), 205 m, 4.XI.2014, leg. Scalercio; 1 male, Saracena (Cosenza), 1010 m, 14.X.2015, leg. Scalercio and Infusino; 1 male, Alessandria del Carretto (Cosenza), 1285 m, 18.IX.2017, leg. Scalercio and Infusino.

Chorotype: Turanic-European. 


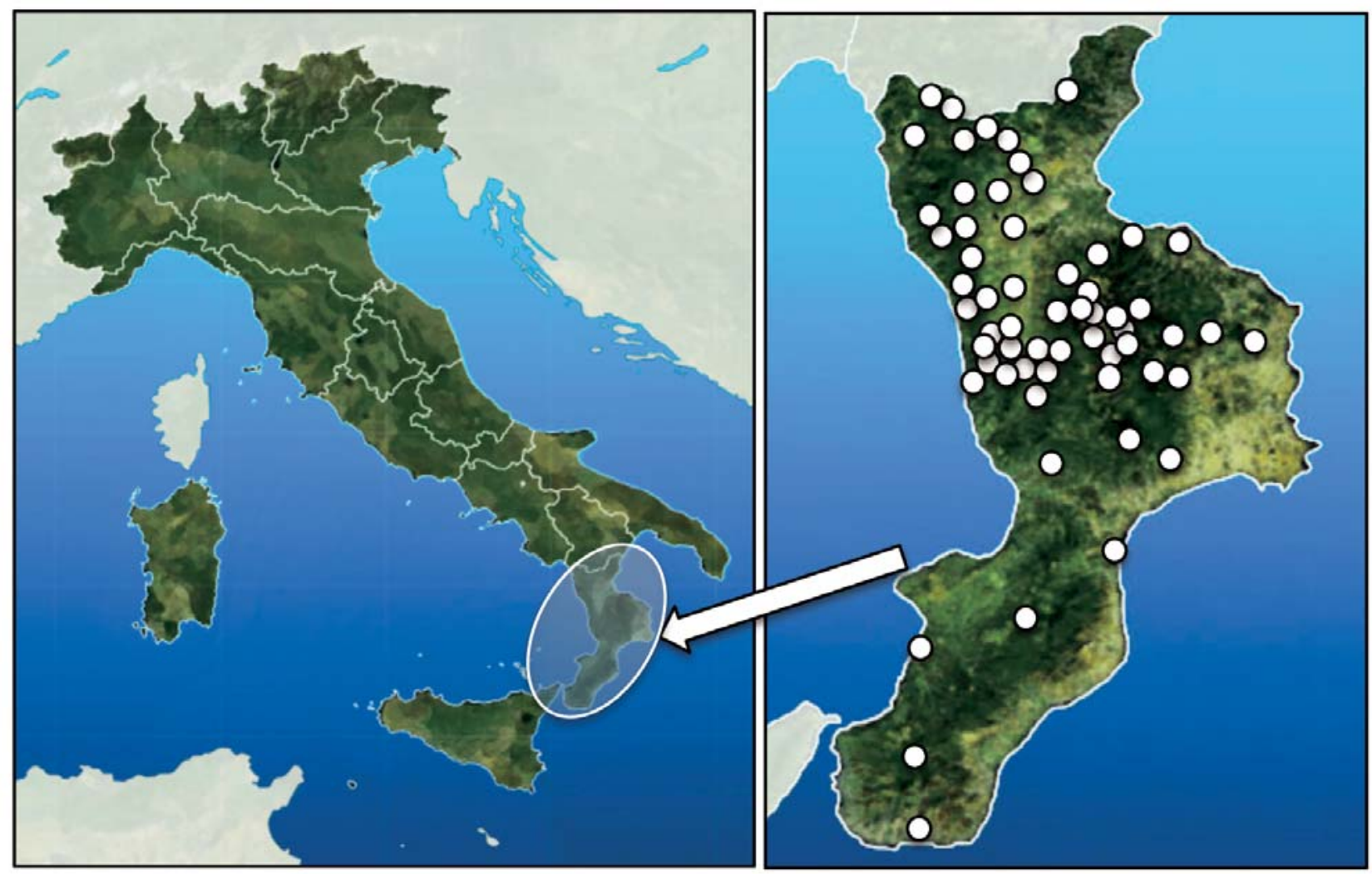

Fig. I - Calabria territory (southern Italy) (on left) and trapping localities (white circles) (on right).

Acleris schalleriana Linnaeus, 1761

Material examined: 1 female, Saracena (Cosenza), 1010 m, 19.VI.2015, leg. Scalercio.

Chorotype: Holarctic.

Acleris cristana ([Denis and Schiffermüller], 1775)

Material examined: 1 male, Aprigliano (Cosenza), 1310 m, 18.V.2015, leg. Scalercio and Infusino; 1 male, Saracena (Cosenza), $1465 \mathrm{~m}, 28$. VII.2015, leg. Scalercio and Infusino; 1 male, Alessandria del Carretto (Cosenza), 1345 m, 17.VIII.2017, leg. Scalercio and Infusino.

Chorotype: Asiatic-European.

Acleris variegana ([Denis and Schiffermüller], 1775)

Material examined: 1 male, Lago Cecita (Cosenza), 1170 m, 15.XI.2013, leg. Scalercio; 1 male, Palizzi Marina (Reggio Calabria), 3 m, 15.V.2015, leg. Urso; 1 female, Fiego di San Fili (Cosenza), 740 m, 28.V.2015, leg. Scalercio and Infusino; 1 male, Saracena (Cosenza), 1010 m, 19.VI.2015, leg. Scalercio and Infusino; 1 female, S.S. Bruno (Vibo Valentia), 1120 m, 22.VI.2015, leg. Scalercio and Infusino; 1 male, Saracena (Cosenza), $1035 \mathrm{~m}$, 23.IX.2015, leg. Scalercio and Infusino; 1 male, Montagna Grande (Cosenza), 1325 m, 4.XI.2015, leg. Scalercio and Infusino; 1 female, S.B. Ullano (Cosenza), $810 \mathrm{~m}$, 1.VI.2016, leg. Scalercio and Infusino; 1 female, Lungro (Cosenza), 1270 m, 6.VI.2016, leg. Scalercio and Infusino.

Chorotype: Holarctic.

Acleris aspersana (Wood, 1839)

Material examined: 1 male, Righio (Cosenza), 1330 m, 24.VII.2017, leg. Scalercio and Infusino.

Chorotype: Centro Asiatic-European.
Acleris hastiana (Linnaeus, 1758)

Material examined: 1 female, S.S. Bruno (Vibo Valentia), $860 \mathrm{~m}$, 7.VII.2016, leg. Scalercio and Infusino.

Chorotype: Holarctic.

Acleris notana (Dunovan, 1806)

Material examined: 1 female, M.te Pettinascura (Cosenza), 1300 m, 11.VI.1991, leg. Trematerra.

Chorotype: Palaearctic.

Acleris ferrugana ([Denis and Schiffermüller], 1775)

Material examined: 1 female, S.S. Bruno (Vibo Valentia), 830 m, 6.IV.2016, leg. Scalercio and Infusino.

Chorotype: Palaearctic.

Remarks: collected on early April, previously reported in TREMATERRA (2003) from June to November.

Acleris quercinana (Zeller, 1849)

Material examined: 1 female, Montalto Uffugo (Cosenza), 545 m, 1.IV.2016, leg. Scalercio and Infusino.

Chorotype: Turanic-European.

* Acleris boscanoides Razowski, 1959

Records: Strongoli (Crotone), 14.VI.1985 (in TREMATERRA, 2003; Pettersson, pers. comm.).

Chorotype: Turanic-European.

Acleris literana (Linnaeus, 1758)

Material examined: 2 females, Alessandria del Carretto (Cosenza), 1345 m, 17.VIII.2017, leg. Scalercio and Infusino.

Chorotype: European. 

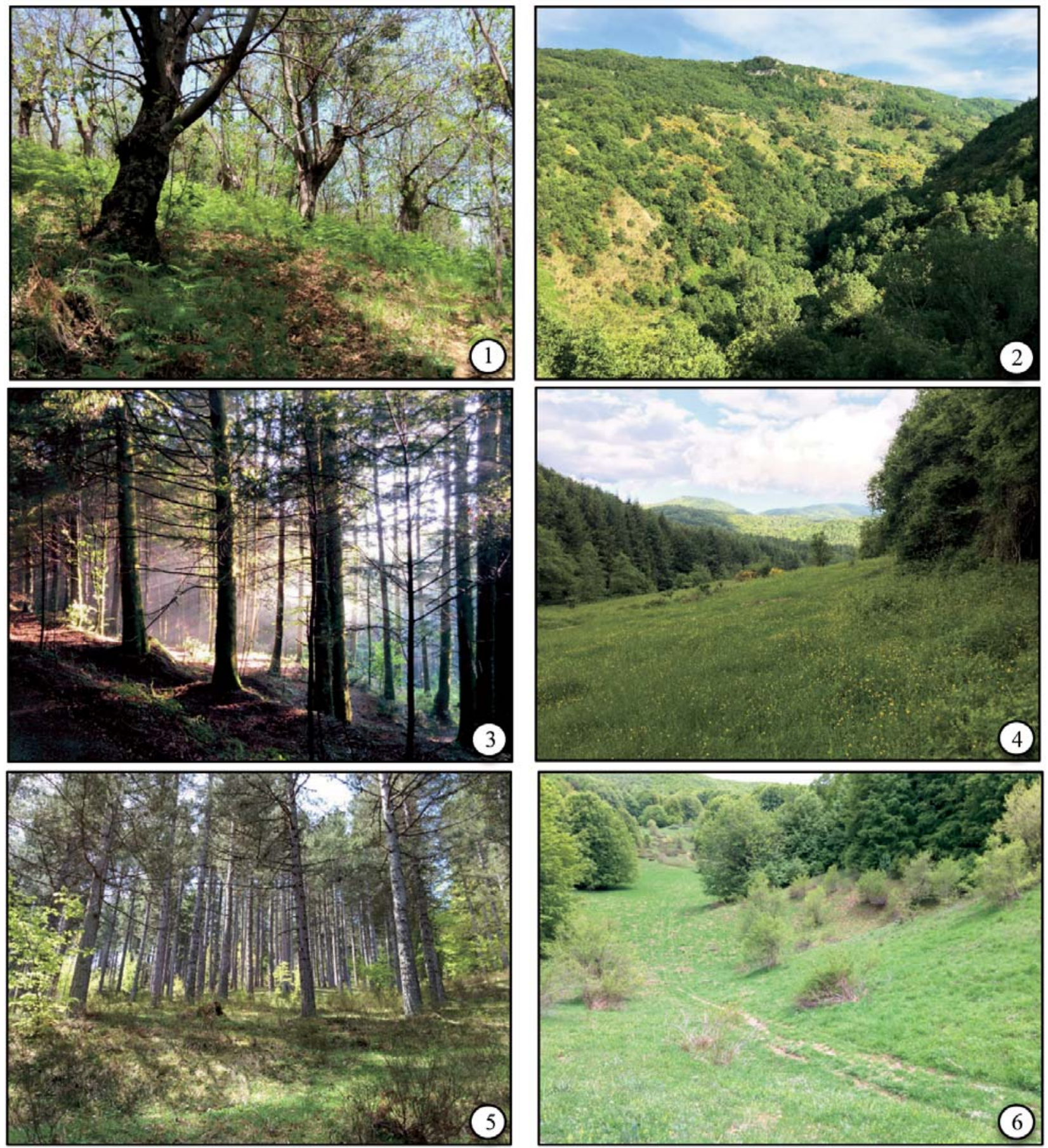

Fig. II - Some habitats visited during entomological expeditions in Calabria territory (southern Italy): Coastal chain of Paola $541 \mathrm{~m}$ a.s.1. (1); Carolei (Coastal chain) 558 m a.s.l. (2); Abetina of Serra San Bruno 917 m a.s.l. (3); Falconara Albanese (Coastal chain) $998 \mathrm{~m}$ a.s.1. (4); Sila National Park 1300 m a.s.l. (5); Orsomarso mountains, Pollino National Park 1470 m a.s.l. (6).

Tribe Cochylini

Phtheochroa purana (Guenée, 1846)

Material examined: 1 male, Vallone Colloreto (Cosenza), 800 m, 6.VI.1981, leg. Scalercio.

Chorotype: S-European.

Phtheochroa duponchelana (Duponchel, 1843)

Material examined: 2 males, Copanello (Catanzaro), 70 m, 9.V.1996, leg. Scalercio; 1 male, Copanello (Catanzaro), 80 m, 24.V.1996, leg. Gatti.

Chorotype: Mediterranean.
Phtheochroa rugosana (Hübner, [1769-1799])

Material examined: 1 female, S.B. Ullano (Cosenza), 893 m, 28.VI.2016, leg. Scalercio and Infusino.

Chorotype: Turanic-European-Mediterranean.

Cochylimorpha sparsana (Staudinger, 1880)

Material examined: 2 males, Vivaio Sbanditi (Cosenza), 1351 $\mathrm{m}, 18$. V.2015, leg. Scalercio and Infusino; 1 male, Pedace (Cosenza), 1450 m, 18.V.2015, leg. Scalercio and Infusino; 1 male, Vivaio Sbanditi (Cosenza), 1351 m, 25.V.2015, leg. Scalercio and Infusino; 1 male, Montagna Grande (Cosenza), 1355 m, 18.V.2016, leg. Scalercio and Infusino.

Chorotype: Mediterranean. 
Table 1 - List of trapping localities grouped by provinces of Cosenza, Crotone, Catanzaro, Vibo Valentia and Reggio Calabria (Calabria region, southern Italy).

\begin{tabular}{|l|l|l|}
\hline \hline Province of Cosenza & Mormanno & Spezzano della Sila, loc. Lago Cecita \\
\hline Acquaformosa & Mormanno, loc. Monte Cerviero & Spezzano della Sila, loc. Vivaio Sbanditi \\
\hline Aieta & Orsomarso & Spezzano Piccolo, loc. Righio \\
\hline Alessandria del Carretto & Orsomarso, loc. Fiume Argentino & \\
\hline Aprigliano & Paola & Province of Crotone \\
\hline Calopezzati & Pedace & Savelli \\
\hline Camigliatello & Rende & Strongoli \\
\hline Camigliatello, loc. Monte Scuro & Rossano, loc. Rossano lido & \\
\hline Campotenese, loc. Cozzi dell'Anticristo & San Benedetto Ullano & Province of Catanzaro \\
\hline Carolei & San Bernardo in Fiore, loc. San Bernardo & Copanello \\
\hline Castrovillari & San Fili & Perticaro \\
\hline Cosenza, loc. Donnici & San Fili, loc. Fiego di San Fili & Sersale \\
\hline Cotronei, loc. Lago Ampollino & San Giovanni in Fiore & Soveria Mannelli \\
\hline Dipignano, loc. Pianette di Dipignano & San Giovanni in Fiore, loc. Mangiatoie & Taverna, loc. Monte Gariglione \\
\hline Falconara Albanese & San Giovanni in Fiore, loc. Montagna Grande & \\
\hline Laino Borgo, loc. Fiume Lao & San Giovanni in Fiore, loc. Monte Pettinascura & Province of Vibo Valentia \\
\hline Longobucco & San Giovanni in Fiore, loc. Rovale & Serra San Bruno \\
\hline Longobucco, loc. La Fossiata & San Lucido & \\
\hline Lungro & San Nicola Silano & Province of Reggio Calabria \\
\hline Mendicino & Sangineto & Palizzi Marina \\
\hline Montalto Uffugo & Saracena & Roccaforte del Greco, loc. Diga del Menta \\
\hline Morano Calabro, loc. Colloreto & Spezzano della Sila & San Ferdinando \\
\hline Morano Calabro, loc. Vallone Colloreto & Spezzano della Sila, loc. Fallistro & \\
\hline
\end{tabular}

Phalonidia gilvicomana (Zeller, 1847)

Material examined: 1 male, S.S. Bruno (Vibo Valentia), $830 \mathrm{~m}, 30 . \mathrm{V} .2016$, leg. Scalercio and Infusino.

Chorotype: European.

Phalonidia manniana (Fischer von Röslerstamm, 1839)

Material examined: 2 males, Orsomarso (Cosenza), 160 m, 27.VIII.2014, leg. Scalercio.

Chorotype: Asiatic-European.

Agapeta hamana (Linnaeus, 1758)

Material examined: 2 males, San Fili (Cosenza), $831 \mathrm{~m}$, 25.V.2017, leg. Colacci, Goglia and Scalercio; 1 male; Falconara Albanese (Cosenza), 921 m, 25.V.2017, leg. Colacci, Goglia and Scalercio.

Chorotype: Centro Asiatic-European.

\section{Agapeta zoegana (Linnaeus, 1767)}

Material examined: 1 male, Pianette di Dipignano (Cosenza), 765 m, 29.VII.2013, leg. Scalercio; 1 male, La Fossiata (Cosenza), 1300 m, 13.VIII.2013, leg. Scalercio; 1 female, Spezzano della Sila (Cosenza), 1350 m, 8.VII.2014, leg. Scalercio; 2 males, Mangiatoie (Cosenza), 1275 m, 25.VII.2016, leg. Scalercio and Infusino.

Chorotype: Turanic-European.

Eupoecilia angustana (Hübner, [1796-1799])

Material examined: 1 female, Alessandria del Carretto (Cosenza), 1337 m, 19.VII.2017, leg. Scalercio and Infusino.

Chorotype: Asiatic-European.
Aethes piercei Obraztsov, 1952

Material examined: 1 male, Cozzi dell'Anticristo (Cosenza), 1300 m, 28.V.1989, leg. Trematerra; 1 female, M.te Crasta (Cosenza), 1200 m, 28.V.1989, leg. Trematerra; 2 females, S. Coppola di Paola (Cosenza), 1400 m, 13.VI.1991, leg. Trematerra.

Chorotype: European.

Aethes williana (Brahm, 1791)

Material examined: 1 male, San Ferdinando (Reggio Calabria), 5 m, 15.VII.2017, leg. Bonelli.

Chorotype: Centro Asiatic-European.

Aethes moribundana (Staudinger, 1859)

Material examined: 5 males, Saracena (Cosenza), 990 m, 20.V.2015, leg. Scalercio and Infusino.

Chorotype: Centro Asiatic-European-Mediterranean.

Aethes tesserana ([Denis and Schiffermüller], 1775)

Material examined: 1 male, Pedace (Cosenza), $1440 \mathrm{~m}$, 15.VI.2015, leg. Scalercio and Infusino; 2 males, San Fili (Cosenza), 831 m, 25.V.2017, leg. Colacci, Goglia and Scalercio.

Chorotype: Centro Asiatic-European.

* Aethes kasyi Razowski, 1962

Records: Cozzi dell'Anticristo (Cosenza), 30.VII.1990, leg. Trematerra (in TREMATERRA et al., 1994).

Chorotype: Turanic-European. 
* Aethes francillana (Fabricius, 1794)

Records: 1 male, Castrovillari (CS), 350 m, 10.VI.1978 (in TrematerRa, 2003; Parenzan, pers. comm.).

Chorotype: W-Palaearctic.

Aethes bilbaensis (Rössler, 1877)

Material examined: 1 male, Donnici (Cosenza), $550 \mathrm{~m}$, 23.VII.2013, leg. Scalercio and Infusino; 1 male, Montagna Grande (Cosenza), 1355 m, 5.VII.2016, leg. Scalercio and Infusino; 1 male, Montalto Uffugo (Cosenza), $550 \mathrm{~m}$, 27.VII.2016, leg. Scalercio and Infusino; 1 male, San Ferdinando (Reggio Calabria), 5 m, 11.VII.2017, leg. Bonelli.

Chorotype: Centro Asiatic-European-Mediterranean.

Aethes rubiginana (Walsingham, 1903)

Material examined: 2 males, Copanello (Catanzaro), 70 m, 10.V.1996, leg. Scalercio; 1 female, Copanello (Catanzaro), 70 m, 10.V.1996, leg. Gatti; 1 female, Copanello (Catanzaro), 70 m, 25.VI.1996, leg. Gatti.

Chorotype: NW-African (extension S-ApenninicSicilian).

Aethes mauritanica (Walsingham, 1898)

Material examined: 1 female, Rossano lido (Cosenza), 8.V.1981, leg. Scalercio.

Chorotype: Mediterranean.

Aethes cnicana (Westwood, 1854)

Material examined: 2 males, S.S. Bruno (Vibo Valentia), 925 m, 7.VII.2016, leg. Scalercio and Infusino.

Chorotype: Asiatic-European.

* Cochylis hybridella (Hübner, [1810-1813])

Records: 3 males, Camigliatello (Cosenza), 3.VIII.1921 (in TREMATERRA, 1991b).

Chorotype: Asiatic-European.

Cochylis salebrana (Mann, 1862)

Material examined: 1 male, Pianette di Dipignano (Cosenza), 765 m, 5.VIII.2013, leg. Scalercio; 1 male, Montalto Uffugo (Cosenza), $550 \mathrm{~m}, 25 . V I .2015$, leg. Scalercio and Infusino.

Chorotype: S-European.

Cochylis molliculana Zeller, 1847

Material examined: 1 male, Rende (Cosenza), 204 m, 24.V.2017, leg. Colacci and Goglia.

Chorotype: S-European.

Cochylis posterana Zeller, 1847

Material examined: 1 male, Lago Cecita (Cosenza), 1176 m, 2.IX.2013, leg. Scalercio; 2 males, San Fili (Cosenza), 630 m, 25.VI.2015, leg. Scalercio and Infusino; 1 male, Fiego di San Fili (Cosenza), 720 m, 25.VI.2015, leg. Scalercio and Infusino; 1 male, Fiego di San Fili (Cosenza), 740 m, 25.VI.2015, leg. Scalercio and Infusino; 1 female, San Bernardo (Cosenza), 1380 m, 11.V.2016, leg. Scalercio and Infusino; 1 male, Saracena (Cosenza), 1420 m, 6.VI.2016, leg. Scalercio and Infusino; 1 female, San Bernardo (Cosenza), $1380 \mathrm{~m}, 25$. VII.2016, leg. Scalercio and Infusino; 1 female, Saracena (Cosenza), 1475 m, 1.VIII.2016, leg. Scalercio and Infusino; 1 female, Montalto Uffugo (Cosenza), $545 \mathrm{~m}$, 29.VIII.2016, leg. Scalercio and Infusino; 1 male, Alessandria del Carretto (Cosenza), 1291 m, 21.VI.2017, leg. Scalercio and Infusino; 1 male, Alessandria del Carretto (Cosenza), $1291 \mathrm{~m}, 19$. VII.2017, leg. Scalercio and Infusino.

Chorotype: W-Palaearctic.
Tribe Cnephasiini

* Propiromorpha rhodophana (Herrich-Schäffer, 1849)

Records: Rossano Lido (Cosenza), 5 m, 20.VII.1981, leg. Parenzan (in TREMATERRA et al., 1997).

Chorotype: Palaearctic.

Eana penziana (Hübner, [1796-1799])

Material examined: 1 male, Saracena (Cosenza), 1035 m, 15.VI.2015, leg. Scalercio and Infusino; 1 male, S.S. Bruno (Vibo Valentia), $860 \mathrm{~m}, 22$. VI.2015, leg. Scalercio and Infusino; 2 males, San Bernardo (Cosenza), $1383 \mathrm{~m}$, 7.VI.2016, leg. Scalercio and Infusino.

Chorotype: Centro Asiatic-European.

Eana italica (Obraztsov, 1950)

Material examined: 1 female, Saracena (Cosenza), 1475 m, 1.VII.2016, leg. Scalercio and Infusino.

Chorotype: Apenninic-Dinaric.

Archicnephasia hartigi Razowski, 1983

Material examined: 1 male, Alessandria del Carretto (Cosenza), 1345 m, 12.XI.2017, leg. Scalercio and Infusino. Chorotype: S-Apenninic.

Cnephasia incertana (Treitschke, 1835)

Material examined: 5 males, Falconara Albanese (Cosenza), 921 m, 25.V.2017, leg. Colacci, Goglia and Scalercio; 5 males, San Fili (Cosenza), 831 m, 25.V.2017, leg. Colacci, Goglia and Scalercio.

Chorotype: W-Palaearctic.

Cnephasia stephensiana (Doubleday, 1850)

Material examined: 1 female, Spezzano della Sila (Cosenza), 1299 m, 24.VII.2017, leg. Scalercio and Infusino; 1 female, Fallistro (Cosenza), $1376 \mathrm{~m}$, 24.VII.2017, leg. Scalercio and Infusino.

Chorotype: Palaearctic.

* Cnephasia asseclana ([Denis and Schiffermüller], 1775)

Records: Sersale (Catanzaro), 6-20.VII.1986 (in

TREMATERRA et al., 1994).

Chorotype: Holarctic.

Cnephasia zangheriana Trematerra, 1991

Material examined: 1 male, Vivaio Sbanditi (Cosenza), 1350 m, 26.VI.2014, leg. Scalercio; 1 male, Saracena (Cosenza), 1370 m, 20.V.2015, leg. Scalercio and Infusino; 2 males, Saracena (Cosenza), 1431 m, 6.VI.2016, leg. Scalercio and Infusino; 3 males, San Nicola Silano (Cosenza), 1435 m, 17.V.2018, leg. Colacci and Goglia.

Chorotype: Apenninic.

Cnephasia pasiuana (Hübner, [1796-1799])

Material examined: 1 female, Palizzi Marina (Reggio Calabria), 3 m, 15.V.2015, leg. Urso.

Chorotype: W-Palaearctic.

Cnephasia communana (Herrich-Schäffer, 1847)

Material examined: 1 female, Pedace (Cosenza), $1450 \mathrm{~m}$, 15.VII.2015, leg. Scalercio and Infusino; 1 male, Acquaformosa (Cosenza), $1357 \mathrm{~m}, 15 . I V .2016$, leg. Scalercio and Infusino; 1 male, Montalto Uffugo (Cosenza), $565 \mathrm{~m}, 1$. VI.2016, leg. Scalercio and Infusino.

Chorotype: Centro Asiatic-European-Mediterranean.

Cnephasia cupressivorana (Staudinger, 1871)

Material examined: 1 male, Alessandria del Carretto (Cosenza), 1253 m, 18.IV.2017, leg. Scalercio and Infusino.

Chorotype: S-European. 
Cnephasia abrasana (Duponchel, 1843) Material examined: 1 male, Righio (CS), $1341 \mathrm{~m}$, 24.V.2017, leg. Scalercio and Infusino; 1 male, Fallistro (Cosenza), 1376 m, 31.V.2017, leg. Scalercio and Infusino.

Chorotype: European.

* Cnephasia chrysantheana (Duponchel, 1842)

Records: Sersale (Catanzaro), 15.VI-20.VII.1985; Sersale (Catanzaro), 15.VI-20.VII.1986 (in TREMATERRA et al., 1994).

Chorotype: W-Palaearctic.

Cnephasia fulturata Rebel, 1940

Material examined: 1 male, Palizzi Marina (Reggio Calabria), 3 m, 15.V.2015, leg. Urso.

Chorotype: Turanic-European-Mediterranean.

Cnephasia hellenica Obraztsov, 1956 Material examined: 1 male, Fiego di San Fili (Cosenza), 740 m, 25.VI.2015, leg. Scalercio and Infusino.

Chorotype: Turanic-European.

Cnephasia ecullyana Réal, 1951

Material examined: 1 female, Rende (Cosenza), 205 m, 14.V.2014, leg. Scalercio.

Chorotype: S-European.

\section{Tribe Archipini}

Batodes angustiorana (Haworth, [1811]) Material examined: 1 male, Montalto Uffugo (Cosenza), 565 m, 1.VI.2016, leg. Scalercio and Infusino.

Chorotype: W-Palaearctic.

Epagoge grotiana (Fabricius, 1781)

Material examined: 1 female, S.B. Ullano (Cosenza), 880 m, 28.VI.2016, leg. Scalercio and Infusino.

Chorotype: W-Palaearctic.

Paramesia gnomana (Clerck, 1759)

Material examined: 1 male, Cozzi dell'Anticristo (Cosenza), 1300 m, 30.VII.1990, leg. Trematerra.

Chorotype: European.

\section{Paramesia diffusana (Kennel, 1899)}

Material examined: 2 males, Lago Cecita (Cosenza), 1163 m, 13.VIII.2013, leg. Scalercio; 1 male, Vivaio Sbanditi (Cosenza), 1350 m, 5.VIII.2014, leg. Scalercio; 1 male, Vivaio Sbanditi (Cosenza), 1350 m, 13.VIII.2014, leg. Scalercio; 1 female, S.S. Bruno (Vibo Valentia), 924 m, 6.IV.2016, leg. Scalercio and Infusino; 1 male, Saracena (Cosenza), 1460 m, 1.VIII.2016, leg. Scalercio and Infusino.

Chorotype: W-Mediterranean.

\section{Archips podanus (Scopoli, 1763)}

Material examined: 2 males, Orsomarso (Cosenza), 160 m, 27.VIII.2014, leg. Scalercio; 1 male, S.S. Bruno (Vibo Valentia), 840 m, 22.VI.2015, leg. Scalercio and Infusino; 1 male, S.S. Bruno (Vibo Valentia), 830 m, 30.V.2016, leg. Scalercio and Infusino.

Chorotype: Asiatic-European.

Archips crataeganus (Hübner, [1796-1799])

Material examined: 1 male, Montalto Uffugo (Cosenza), 230 m, 24.V.2014, leg. Scalercio.

Chorotype: Palaearctic.
Archips rosanus (Linnaeus, 1758)

Material examined: 1 male, Fiego di San Fili (Cosenza), 720 m, 25.VI.2015, leg. Scalercio and Infusino.

Chorotype: Palaearctic.

Choristoneura diversana (Hübner, [1814-1817])

Material examined: 1 male, S.S. Bruno (Vibo Valentia), 840 m, 22.VI.2015, leg. Scalercio and Infusino.

Chorotype: Asiatic-European.

* Choristoneura murinana (Hübner, [1796-1799])

Records: Pollino, 10.VII.1989, leg. Trematerra (in TREMATERRA et al., 1994).

Chorotype: Asiatic-European.

Argyrotaenia ljungiana (Thunberg, 1797)

Material examined: 1 male, Montalto Uffugo (Cosenza), 545 m, 25.VI.2015, leg. Scalercio and Infusino.

Chorotype: Holarctic.

Ptycoloma lecheana (Linnaeus, 1758)

Material examined: 1 male, Alessandria del Carretto (Cosenza), 1285 m, 21.VI.2017, leg. Scalercio and Infusino. Chorotype: Asiatic-European.

Pandemis cerasana (Hübner, 1786)

Material examined: 1 male, Vivaio Sbanditi (Cosenza), 1350 m, 26.VI.2014, leg. Scalercio; 1 male, Vivaio Sbanditi (Cosenza), 1350 m, 29.VII.2014, leg. Scalercio.

Chorotype: Asiatic-European.

Pandemis heparana ([Denis and Schiffermüller], 1775)

Material examined: 1 female, Lago Cecita (Cosenza), 1163 m, 30.VII.2013, leg. Scalercio; 2 males, La Fossiata (Cosenza), 1300 m, 13.VIII.2013, leg. Scalercio; 1 female, Vivaio Sbanditi (Cosenza), 1350 m, 29.VII.2014, leg. Scalercio; 2 males, Lago Cecita (Cosenza), $1163 \mathrm{~m}$, 13.VIII.2014, leg. Scalercio; 2 males, Orsomarso (Cosenza), 160 m, 27.VIII.2014, leg. Scalercio.

Chorotype: Asiatic-European.

Syndemis musculana (Hübner, [1796-1799])

Material examined: 1 male, Vivaio Sbanditi (Cosenza), 1350 m, 26.VI.2014, leg. Scalercio; 1 male, Vivaio Sbanditi (Cosenza), 1350 m, 20.X.2014, leg. Scalercio; 1 female, S.S. Bruno (Vibo Valentia), 830 m, 30.V.2016, leg. Scalercio and Infusino; 1 male, S.S. Bruno (Vibo Valentia), 835 m, 30.V.2016, leg. Scalercio and Infusino; 1 male, S.S. Bruno (Vibo Valentia), 883 m, 30.V.2016, leg. Scalercio and Infusino; 1 male, S.S. Bruno (Vibo Valentia), 917 m, 30.V.2016, leg. Scalercio and Infusino; 1 male, S.S. Bruno (Vibo Valentia), 924 m, 30.V.2016, leg. Scalercio and Infusino; 1 male, S.B. Ullano (Cosenza), 810 m, 1.VI.2016, leg. Scalercio and Infusino; 1 female, S.B. Ullano (Cosenza), 880 m, 1.VI.2016, leg. Scalercio and Infusino; 1 female, Saracena (Cosenza), $1431 \mathrm{~m}, 6 . V I .2016$, leg. Scalercio and Infusino; 1 male, San Giovanni in Fiore (Cosenza), 1250 m, 24.V.2017, leg. Scalercio and Infusino; 2 males, San Fili (Cosenza), 831 m, 25.V.2017, leg. Colacci, Goglia and Scalercio; 1 male, Rovale (Cosenza), 1440 m, 17.V.2018, leg. Colacci and Goglia.

Chorotype: Asiatic-European.

Cacoecimorpha pronubana (Hübner, [1796-1799])

Material examined: 1 male, Montalto Uffugo (Cosenza), 
550 m, 27.VII.2016, leg. Scalercio and Infusino; 1 female, San Ferdinando (Reggio Calabria), 5 m, 15.VII.2017, leg. Bonelli.

Chorotype: European-Mediterranean.

*Aphelia ochreana (Hübner, [1797-1799])

Records: Cozzo San Lorenzo (Cosenza), 29.V.1990 (in TREMATERRA et al., 1994).

Chorotype: Turanic-European.

Dichelia histrionana (Frölich, 1828)

Material examined: 1 male, Vivaio Sbanditi (Cosenza), 1355 m, 18.VI.2014, leg. Scalercio; 2 males, Vivaio Sbanditi (Cosenza), 1350 m, 20.VII.2014, leg. Scalercio; 1 male, Vivaio Sbanditi (Cosenza), 1351 m, 27.VII.2015, leg. Scalercio and Infusino.

Chorotype: European.

* Clepsis spectrana (Treitschke, 1830)

Records: Rossano (Cosenza), 2 m, 8.V.1981, leg. Parenzan (in TrEMATERRA et al., 1997).

Chorotype: European.

* Clepsis pallidana (Fabricius, 1776)

Records: Rossano (Cosenza), 2 m, 8.V.1981, leg. Parenzan (in TREMATERRA et al., 1997).

Chorotype: Asiatic-European.

Clepsis consimilana (Hübner, [1814-1817])

Material examined: 1 female, Donnici (Cosenza), $550 \mathrm{~m}$, 23.VII.2013, leg. Scalercio; 1 male, Pianette di Dipignano (Cosenza), 765 m, 5.VIII.2013, leg. Scalercio; 1 male, Rende (Cosenza), 205 m, 14.V.2014, leg. Scalercio; 1 male, Sangineto (Cosenza), 50 m, 10.VIII.2014, leg. Scalercio; 1 male, Palizzi Marina (Reggio Calabria), 3 m, 15.V.2015, leg. Urso; 1 male, Montalto Uffugo (Cosenza), $565 \mathrm{~m}$, 28.V.2015, leg. Scalercio and Infusino; 1 male, Rende (Cosenza), 204 m, 24.V.2017, leg. Colacci and Goglia; 4 males, Carolei (Cosenza), 558 m, 24.V.2017, leg. Colacci, Goglia and Scalercio; 1 male and 1 female, Paola (Cosenza), 2 m, 25.V.2017, leg. Colacci, Goglia and Scalercio; 3 males, San Fili (Cosenza), 831 m, 25.V.2017, leg. Colacci, Goglia and Scalercio.

Chorotype: W-Palaearctic.

Tribe Euliini

* Eulia ministrana (Linnaeus, 1758)

Records: Sersale (Catanzaro), 29.VI-3.VIII.1985; Sersale (Catanzaro), 29.VI-3.VIII.1986 (in TREMATERRA et al., 1994).

Chorotype: Holarctic.

\section{SUBFAMILY CHLIDANOTINAE}

Tribe Polyorthini

Olindia schumacherana (Fabricius, 1787)

Material examined: 1 female, S.S. Bruno (Vibo Valentia), $860 \mathrm{~m}, 22$. VI.2015, leg. Scalercio and Infusino; 2 males, S.S. Bruno (Vibo Valentia), 924 m, 30.V.2016, leg. Scalercio and Infusino.

Chorotype: European.
Isotrias rectifasciana (Haworth, [1811])

Material examined: 1 female, Lago Cecita (Cosenza), 1163 m, 30.VII.2013, leg. Scalercio.

Chorotype: European.

* Isotrias joannisana (Turati, 1921)

Records: Cozzi dell'Anticristo (Cosenza), 28.V.1989; Cozzi dell'Anticristo (Cosenza), 28.V.1990 (in TREMATERRA et al., 1994).

Chorotype: Apenninic.

Isotrias martelliana Trematerra, 1990

Material examined: 1 male and 1 female, Cozzi dell'Anticristo (Cosenza), 1300 m, 28.V.1989, leg. Trematerra.

Chorotype: S-Apenninic.

\section{SUBFAMILY OLETHREUTINAE}

Tribe Bactrini

Bactra bactrana (Kennel, 1901)

Material examined: 1 male, Righio (Cosenza), 1355 m, 23.V.2014, leg. Scalercio; 1 male, S.S. Bruno (Vibo Valentia), 830 m, 10.V.2016, leg. Scalercio and Infusino.

Chorotype: Subcosmopolitan.

Bactra lancealana (Hübner, [1796-1799])

Material examined: 1 female, Orsomarso (Cosenza), 160 m, 27.VIII.2014, leg. Scalercio.

Chorotype: Subcosmopolitan.

Bactra venosana (Zeller, 1847)

Material examined: 1 male, Paola (Cosenza), $20 \mathrm{~m}$, 9.VI.2017, leg. Scalercio.

Chorotype: Subcosmopolitan.

Tribe Olethreutini

Endothenia gentianaeana (Hübner, [1796-1799])

Material examined: 1 male and 1 female, Orsomarso (Cosenza), 160 m, 27.VIII.2014, leg. Scalercio.

Chorotype: Asiatic-European.

* Endothenia marginana (Haworth, [1811])

Records: 1 male, Aieta (Cosenza), 500 m, 12.VII.1989, leg. Hausmann (in TrEMATERRA, 2003; unpubl. data).

Chorotype: Palaearctic.

Endothenia lapideana (Herrich-Schäffer, 1848)

Material examined: 1 male, Aieta (Cosenza), $500 \mathrm{~m}$, 12.VII.1989, leg. Hausmann.

Chorotype: European.

Endothenia nigricostana (Haworth, [1811])

Material examined: 1 male, Vivaio Sbanditi (Cosenza), 1350 m, 13.VIII.2014, leg. Scalercio.

Chorotype: Asiatic-European.

Endothenia pullana (Haworth, [1811])

Material examined: 1 female, Lago Cecita (Cosenza), 1163 m, 13.VIII.2014, leg. Scalercio.

Chorotype: Sibiric-European. 
Lobesia botrana ([Denis and Schiffermüller], 1775)

Material examined: 1 male, Calopezzati (Cosenza), $10 \mathrm{~m}$, 25.IV.2015, leg. Scalercio.

Chorotype: Holarctic.

* Lobesia bicinctana (Duponchel, 1844)

Records: 1 male, Aieta (Cosenza), 300 m, 11.VII.1989, leg. Hausmann (in TrEMATERRA, 2003; unpubl. data). Chorotype: Holarctic.

Eudemis profundana ([Denis and Schiffermüller], 1775) Material examined: 1 male, Donnici (Cosenza), 550 m, 23.VII.2013, leg. Scalercio.

Chorotype: European.

* Hedya salicella (Linnaeus, 1758)

Records: 1 female, Lao river (Cosenza), 50 m, 8.VI.1990, leg. Hausmann (in TREMATERRA, 2003; unpubl. data).

Chorotype: Asiatic-European.

Hedya nubiferana (Haworth, [1811])

Material examined: 1 male, Alessandria del Carretto (Cosenza), 1285 m, 21.VI.2017, leg. Scalercio and Infusino. Chorotype: Centro Asiatic-European.

* Hedya ochroleucana (Frölich, 1828) Records: Sersale (Catanzaro), 15.VI-10.VIII.1985 (in TREMATERRA et al., 1994).

Chorotype: Holarctic.

Metendothenia atropunctana (Zetterstedt, 1839)

Material examined: 1 male, Saracena (Cosenza), $1340 \mathrm{~m}$, 20.V.2015, leg. Scalercio and Infusino; 1 male, Vivaio Sbanditi (Cosenza), $1351 \mathrm{~m}, 25 . \mathrm{V} .2015$, leg. Scalercio and Infusino; 1 male, S.S. Bruno (Vibo Valentia), $970 \mathrm{~m}$, 26.V.2015, leg. Scalercio and Infusino; 1 male, Vivaio Sbanditi (Cosenza), 1352 m, 15.VI.2015, leg. Scalercio and Infusino; 1 male, Acquaformosa (Cosenza), $1357 \mathrm{~m}$, 6.VI.2016, leg. Scalercio and Infusino; 1 female, Saracena (Cosenza), $1431 \mathrm{~m}, 6$.VI.2016, leg. Scalercio and Infusino; 1 male, Saracena (Cosenza), $1010 \mathrm{~m}$, leg. Scalercio and Infusino; 1 male, San Nicola Silano (Cosenza), 1435 m, 17.V.2018, leg. Colacci and Goglia.

Chorotype: Holarctic.

Piniphila bifasciana (Haworth, [1811])

Material examined: 1 female, Lago Cecita (Cosenza), 1163 m, 30.VII.2013, leg. Scalercio; 1 male, La Fossiata (Cosenza), 1300 m, 13.VIII.2013, leg. Scalercio; 1 male, Vivaio Sbanditi (Cosenza), 1350 m, 5.VIII.2014, leg. Scalercio; 3 males, Montagna Grande (Cosenza), 1325 m, 25.VII.2016, leg. Scalercio and Infusino.

Chorotype: Asiatic-European.

Olethreutes arcuellus (Clerck, 1759)

Material examined: 2 males, Vivaio Sbanditi (Cosenza), 1350 m, 18.VI.2014, leg. Scalercio; 4 males, San Fili (Cosenza), 831 m, 25.V.2017, leg. Colacci, Goglia and Scalercio; 4 males, Falconara Albanese (Cosenza), $921 \mathrm{~m}$, 25.V.2017, leg. Colacci, Goglia and Scalercio; 1 male, San Nicola Silano (Cosenza), 1435 m, 17.V.2018, leg. Colacci and Goglia.

Chorotype: Centro Asiatic-European.

Celypha cespitana (Hübner, [1814-1817])

Material examined: 1 male, San Bernardo (Cosenza), 1380 m, 25.VII.2016, leg. Scalercio and Infusino.

Chorotype: Holarctic.
Syricoris lacunana ([Denis and Schiffermüller], 1775)

Material examined: 1 male, Donnici (Cosenza), $550 \mathrm{~m}$, 7.VIII.2013, leg. Scalercio; 2 males, Sila (Cosenza), 1300 m, 18.VI.2014, leg. Scalercio; 1 male, Vivaio Sbanditi (Cosenza), 1350 m, 26.VI.2014, leg. Scalercio; 2 males, Vivaio Sbanditi (Cosenza), 1350 m, 17.VII.2014, leg. Scalercio; 2 males, Montalto Uffugo (Cosenza), 540 m, 28.V.2015, leg. Scalercio and Infusino; 1 male, Montagna Grande (Cosenza), 1370 m, 15.VI.2015, leg. Scalercio and Infusino; 2 males, Saracena (Cosenza), 1475 m, 19.VI.2015, leg. Scalercio and Infusino; 2 males, S.S. Bruno (Vibo Valentia), 840 m, 22.VI.2015, leg. Scalercio and Infusino; 1 male, S.S. Bruno (Vibo Valentia), 830 m, 30.V.2016, leg. Scalercio and Infusino; 2 males, S.S. Bruno (Vibo Valentia), 924 m, 30.V.2016, leg. Scalercio and Infusino; 1 male, Lungro (Cosenza), 1270 m, 6.VI.2016, leg. Scalercio and Infusino; 1 male, Acquaformosa (Cosenza), $1366 \mathrm{~m}$, 6.VI.2016, leg. Scalercio and Infusino; 2 males and 1 female, Carolei (Cosenza), 558 m, 24.V.2017, leg. Colacci, Goglia and Scalercio; 9 males and 3 females, San Fili (Cosenza), $831 \mathrm{~m}$, 25.V.2017, leg. Colacci, Goglia and Scalercio; 12 males, Falconara Albanese (Cosenza), 921 m, 25.V.2017, leg. Colacci, Goglia and Scalercio; 1 male, Alessandria del Carretto (Cosenza), 1285 m, 21.VI.2017, leg. Scalercio and Infusino.

Chorotype: Asiatic-European.

Syricoris rivulana (Scopoli, 1763)

Material examined: 1 male, S.S. Bruno (Vibo Valentia), 835 m, 30.V.2016, leg. Scalercio and Infusino.

Chorotype: Asiatic-European.

Tribe Enarmoniini

Ancylis laetana (Fabricius, 1775)

Material examined: 1 male, Soveria Mannelli (Catanzaro), 12.VI.1991, leg. Trematerra.

Chorotype: Sibiric-European.

\section{Ancylis comptana (Frölich, 1828)}

Material examined: 1 male, Saracena (Cosenza), 1315 m, 14.V.2015, leg. Scalercio and Infusino.

Chorotype: Holarctic.

Ancylis paludana (Barrett, 1871)

Material examined: 3 males, San Fili (Cosenza), $831 \mathrm{~m}$, 25.V.2017, leg. Colacci, Goglia and Scalercio.

Chorotype: Holarctic.

Ancylis badiana ([Denis and Schiffermüller], 1775)

Material examined: 1 female, Vivaio Sbanditi (Cosenza), 1350 m, 26.VI.2014, leg. Scalercio; 1 male, Montagna Grande (Cosenza), 1370 m, 15.VI.2015, leg. Scalercio and Infusino.

Chorotype: Asiatic-European.

Ancylis mitterbacheriana ([Denis and Schiffermüller], 1775)

Material examined: 1 male, S.B. Ullano (Cosenza), 893 m, 1.VI.2016, leg. Scalercio and Infusino; 1 male, Saracena (Cosenza), 1431 m, 6.VI.2016, leg. Scalercio and Infusino.

Chorotype: European.

Tribe Eucosmini

* Thiodia major (Rebel, 1903)

Records: Mendicino (Cosenza), 15.VI-6.VII.1989 (in

TREMATERRA et al., 1994).

Chorotype: S-European. 
Thiodia trochilana (Frölich, 1828)

Material examined: 2 males, Saracena (Cosenza), 1035 m, 15.VI.2015, leg. Scalercio and Infusino.

Chorotype: Turanic-European-Mediterranean.

Rhopobota stagnana ([Denis and Schiffermüller], 1775)

Material examined: 2 males, Cozzi dell'Anticristo (Cosenza), 1300 m, 28.V.1989, leg. Trematerra.

Chorotype: European.

Rhopobota naevana (Hübner, [1814-1817])

Material examined: 1 female, S.S. Bruno (Vibo Valentia), 886 m, 30.V.2016, leg. Scalercio and Infusino.

Chorotype: Subcosmopolitan.

Spilonota ocellana ([Denis and Schiffermüller], 1775)

Material examined: 1 female, Lago Cecita (Cosenza), 1163 m, 30.VII.2013, leg. Scalercio; 1 male and 1 female, Vivaio Sbanditi (Cosenza), 1350 m, 29.VII.2014, leg. Scalercio; 1 male, Mormanno (Cosenza), $970 \mathrm{~m}$, 1.VIII.2016, leg. Scalercio and Infusino; 1 female, Acquaformosa (Cosenza), 1357 m, 1.VIII.2016, leg. Scalercio and Infusino; 1 male, S.S. Bruno (Vibo Valentia), 883 m, 3.VIII.2016, leg. Scalercio and Infusino; 1 male, S.S. Bruno (Vibo Valentia), 925 m, 3.VIII.2016, leg. Scalercio and Infusino; 1 male, Alessandria del Carretto (Cosenza), 1253 m, 13.VII.2017, leg. Scalercio and Infusino; 1 male, Alessandria del Carretto (Cosenza), 1345 m, 19.VII.2017, leg. Scalercio and Infusino.

Chorotype: Holarctic.

* Spilonota laricana (Heinemann, 1863)

Records: Sersale (Catanzaro), 20.VII.1985 (in TREMATERRA et al., 1994).

Chorotype: Holarctic.

Epinotia festivana (Hübner, 1799)

Material examined: 1 female, Donnici (Cosenza), $550 \mathrm{~m}$, 23.VII.2013, leg. Scalercio; 1 male, Montalto Uffugo (Cosenza), 550 m, 25.VI.2015, leg. Scalercio and Infusino; 1 male, Montalto Uffugo (Cosenza), 545 m, 1.VI.2016, leg. Scalercio and Infusino.

Chorotype: Holarctic.

Epinotia thapsiana (Zeller, 1847)

Material examined: 1 female, Palizzi Marina (Reggio Calabria), 3 m, 15.V.2015, leg. Urso.

Chorotype: Centro Asiatic-European-Mediterranean.

Epinotia sordidana (Hübner, [1823-1824])

Material examined: 1 male, Vivaio Sbanditi (Cosenza), 1350 m, 13.X.2014, leg. Scalercio; 1 male, Alessandria del Carretto (Cosenza), 1285 m, 18.IX.2017, leg. Scalercio and Infusino; 1 male, Alessandria del Carretto (Cosenza), 1345 m, 18.IX.2017, leg. Scalercio and Infusino; 1 female, Alessandria del Carretto (Cosenza), 1305 m, 12.XI.2017, leg. Scalercio and Infusino; 1 female, Alessandria del Carretto (Cosenza), 1345 m, 12.XI.2017, leg. Scalercio and Infusino.

Chorotype: European.

Epinotia solandriana (Linnaeus, 1758)

Material examined: 1 male, Vivaio Sbanditi (Cosenza), 1350 m, 13.X.2014, leg. Scalercio.

Chorotype: Holarctic.

Remarks: collected in middle October, previously reported in TREMATERRA (2003) from June to August.
Epinotia signatana (Douglas, 1845)

Material examined: 2 females, Cozzi dell'Anticristo (Cosenza), 1000 m, 13.VI.1991, leg. Trematerra.

Chorotype: Asiatic-European.

Epinotia immundana (Ficher von Röslerstamm, 1839)

Material examined: 1 male, Donnici (Cosenza), $550 \mathrm{~m}$, 23.VII.2013, leg. Scalercio.

Chorotype: Turanic-European.

Epinotia subocellana (Donovan, [1806])

Material examined: 1 male, Rovale (Cosenza), 1440 m, 17.V.2018, leg. Colacci and Goglia.

Chorotype: Sibiric-European.

* Epinotia nigricana (Herrich-Schäffer, 1847)

Records: Sila varie località (Cosenza), 13.VI.1991 (in

TREMATERRA et al., 1994).

Chorotype: European.

Epinotia tenerana ([Denis and Schiffermüller], 1775)

Material examined: 2 males, Vivaio Sbanditi (Cosenza), 1350 m, 17.VII.2014, leg. Scalercio; 1 male, Vivaio Sbanditi (Cosenza), 1350 m, 29.VII.2014, leg. Scalercio; 1 female, Vivaio Sbanditi (Cosenza), 1350 m, 13.VIII.2014, leg. Scalercio; 1 male, Vivaio Sbanditi (Cosenza), $1351 \mathrm{~m}$, 6.VII.2015, leg. Scalercio and Infusino; 1 female, Vivaio Sbanditi (Cosenza), $1351 \mathrm{~m}, 27$. VII.2015, leg. Scalercio and Infusino; 1 female, S.S. Bruno (Vibo Valentia), $917 \mathrm{~m}$, 3.VIII.2016, leg. Scalercio and Infusino; 3 females, Lungro (Cosenza), 1270 m, 31.VIII.2016, leg. Scalercio and Infusino; 1 male, San Fili (Cosenza), 831 m, 25.V.2017, leg. Colacci, Goglia and Scalercio; 1 male, Spezzano della Sila (Cosenza), 1324 m, 24.VII.2017, leg. Scalercio and Infusino.

Chorotype: Cosmopolitan.

Epinotia fraternana (Haworth, [1811])

Material examined: 1 female, San Fili (Cosenza), $831 \mathrm{~m}$, 25.V.2017, leg. Colacci, Goglia and Scalercio.

Chorotype: European.

Crocidosema plebejana Zeller, 1847

Material examined: 1 male and 1 female, Montescuro (Cosenza), 1610 m, 6.III.2016, leg. Scalercio; 1 male, Montalto Uffugo (Cosenza), $550 \mathrm{~m}, 1$. VI.2016, leg. Scalercio and Infusino; 1 male, S.B. Ullano (Cosenza), 825 $\mathrm{m}, 1$. VI.2016, leg. Scalercio and Infusino; 1 male, S.B. Ullano (Cosenza), 845 m, 1.VI.2016, leg. Scalercio and Infusino; 1 female, Paola (Cosenza), 20 m, 9.VI.2017, leg. Scalercio; 1 male, Alessandria del Carretto (Cosenza), 1314 m, 21.VI.2017, leg. Scalercio and Infusino.

Chorotype: Cosmopolitan.

Remarks: collected also on early March, previously reported in TREMATERRA (2003) from April to November and in Trematerra and Colacci (2016) from May to November.

Pelochrista mollitana (Zeller, 1847)

Material examined: 2 males, Palizzi Marina (Reggio Calabria), 3 m, 15.V.2015, leg. Urso; 1 male, S.S. Bruno (Vibo Valentia), 883 m, 3.VIII.2016, leg. Scalercio and Infusino.

Chorotype: Turanic-European-Mediterranean.

Pelochrista agrestana (Treitschke, 1830)

Material examined: 1 male, Savelli (Crotone), 1000 m, 12.VIII.1998, leg. Sciarretta.

Chorotype: S-European 
* Pelochrista bleuseana (Oberthür, 1888)

Records: Sersale (Catanzaro), 6.VII.1985; Sersale (Catanzaro), 22.IX.1985; Perticaro (Catanzaro), 6.VII.1988; Mendicino (Cosenza), 4-18.VII.1988; Perticaro (Catanzaro) 18.VIII.1988; Mendicino (Cosenza), 19.VI.1989; Mendicino (Cosenza), 3.X.1989 (in Trematerra et al., 1994).

Chorotype: W-Mediterranean.

Pelochrista subtiliana (Jäckh, 1960)

Material examined: 1 male, San Fili (Cosenza), $831 \mathrm{~m}$, 25.V.2017, leg. Colacci, Goglia and Scalercio; 1 male, Falconara Albanese (Cosenza), 921 m, 25.V.2017, leg. Colacci, Goglia and Scalercio.

Chorotype: Central-European.

Eucosma cana (Haworth, [1811])

Material examined: 1 male, Fossiata (Cosenza), $1300 \mathrm{~m}$, 13.VIII.2013, leg. Scalercio; 1 male, Vivaio Sbanditi (Cosenza), 1350 m, 29.VII.2014, leg. Scalercio; 1 male, S.S. Bruno (Vibo Valentia), 940 m, 19.VII.2015, leg. Scalercio and Infusino; 1 male, Spezzano della Sila, 1299 $\mathrm{m}, 24$.VII.2017, leg. Scalercio and Infusino.

Chorotype: Asiatic-European.

Eucosma hohenwartiana ([Denis and Schiffermüller], 1775) Material examined: 2 males, Pianette di Dipignano (Cosenza), 765 m, 5.VIII.2013, leg. Scalercio.

Chorotype: Centro Asiatic-European-Mediterranean.

Eucosma balatonana (Osthelder, 1937)

Material examined: 1 male, Montagna Grande (Cosenza), 1370 m, 15.VI.2015, leg. Scalercio and Infusino; 1 female, Montalto Uffugo (Cosenza), 545 m, 25.VI.2015, leg. Scalercio and Infusino; 1 male, Vivaio Sbanditi (Cosenza), $1351 \mathrm{~m}, 6$. VII.2015, leg. Scalercio and Infusino.

Chorotype: Centro Asiatic-European.

Eucosma conterminana (Guenée, 1845)

Material examined: 1 male, Cozzi dell'Anticristo (Cosenza), 1000 m, 13.VI.1992, leg. Trematerra.

Chorotype: Asiatic-European.

Eucosma albidulana (Herrich-Schäffer, 1848)

Material examined: 1 male, Pianette di Dipignano (Cosenza), 765 m, 5.VIII.2013, leg. Scalercio; 1 male, Aprigliano (Cosenza), 1310 m, 15.VI.2015, leg. Scalercio and Infusino.

Chorotype: Centro Asiatic-European-Mediterranean.

Gypsonoma minutana (Hübner, [1796-1799])

Material examined: 1 male, San Fili (Cosenza), $831 \mathrm{~m}$, 25.V.2017, leg. Colacci, Goglia and Scalercio.

Chorotype: Palaearctic.

Gypsonoma sociana (Haworth, 1811)

Material examined: 1 male, Cozzi dell'Anticristo, 1300 m, 28.V.1990, leg. Trematerra.

Chorotype: Asiatic-European.

Epiblema sticticanum (Fabricius, 1794)

Material examined: 1 male, San Lucido (Cosenza), 950 m, 25.V.2017, leg. Colacci, Goglia and Scalercio.

Chorotype: Asiatic-European.

* Epiblema scutulanum ([Denis and Schiffermüller], 1775) Records: Sersale (Catanzaro), 3-17.VIII.1985; Sersale
(Catanzaro), 3-17.VIII.1986; Soveria Mannelli (Catanzaro), 4.VI-27.VII.1987; Cozzi dell'Anticristo (Cosenza), 28.V.1989; Monte Gariglione (Catanzaro), 12.VI.1991 (in TREMATERRA et al., 1994).

Chorotype: Palaearctic.

Epiblema foenellum (Linnaeus, 1758)

Material examined: 1 male, Fiego di San Fili (Cosenza), $720 \mathrm{~m}, 22$. VII.2015, leg. Scalercio and Infusino; 2 males, Montalto Uffugo (Cosenza), 545 m, 28.VI.2016, leg. Scalercio and Infusino; 2 females, Montalto Uffugo (Cosenza), 545 m, 29.VIII.2016, leg. Scalercio and Infusino.

Chorotype: Asiatic-European.

Epiblema costipunctana (Haworth, [1811])

Material examined: 1 male, S. Coppola di Paola (Cosenza), 1300 m, 28.V.1990, leg. Trematerra.

Chorotype: European.

Epiblema graphana (Treitschke, 1835)

Material examined: 2 males and 1 female, M.te Gariglione (Catanzaro), 1600 m, 12.VI.1991, leg. Trematerra.

Chorotype: Centro Asiatic-European.

Notocelia cynosbatella (Linnaeus, 1758)

Material examined: 1 female, Vivaio Sbanditi (Cosenza), 1350 m, 26.VI.2014, leg. Scalercio; 1 male, Vivaio Sbanditi (Cosenza), 1350 m, 29.VII.2014, leg. Scalercio; 1 male, Vivaio Sbanditi (Cosenza), 1353 m, 15.VI.2015, leg. Scalercio and Infusino; 1 male, Montagna Grande (Cosenza), 1370 m, 15.VI.2015, leg. Scalercio and Infusino; 1 male, Mangiatoie (Cosenza), 1270 m, 11.V.2016, leg. Scalercio and Infusino; 1 male, Saracena (Cosenza), 1433 m, 6.VI.2016, leg. Scalercio and Infusino; 1 male, Saracena (Cosenza), 1431 m, 6.VI.2016, leg. Scalercio and Infusino; 3 males, San Fili (Cosenza), 831 m, 25.V.2017, leg. Colacci, Goglia and Scalercio; 1 male, Alessandria del Carretto (Cosenza), 1285 m, 21.VI.2017, leg. Scalercio and Infusino; 1 male, San Nicola Silano (Cosenza), 1435 m, 17.V.2018, leg. Colacci and Goglia.

Chorotype: Asiatic-European.

Notocelia uddmanniana (Linnaeus, 1758)

Material examined: 1 male, Vivaio Sbanditi (Cosenza), 1350 m, 24.VII.2014, leg. Scalercio; 1 male, San Fili (Cosenza), 630 m, 28.V.2015, leg. Scalercio and Infusino; 1 male, Fiego di San Fili (Cosenza), 720 m, 28.V.2015, leg. Scalercio and Infusino; 1 male, Aprigliano (Cosenza), 1310 m, 15.VI.2015, leg. Scalercio and Infusino; 1 male, S.S. Bruno (Vibo Valentia), 1080 m, 22.VI.2015, leg. Scalercio and Infusino; 1 male, Fiego di San Fili (Cosenza), $720 \mathrm{~m}$, 25.VI.2015, leg. Scalercio and Infusino; 1 male, S.S. Bruno (Vibo Valentia), 879 m, 10.V.2016, leg. Scalercio and Infusino; 1 male, S.B. Ullano (Cosenza), 825 m, 27.VII.2016, leg. Scalercio and Infusino; 1 female, San Fili (Cosenza), 831 m, 25.V.2017, leg. Colacci, Goglia and Scalercio.

Chorotype: W-Palaearctic.

Notocelia aquana (Hübner, [1796-1799])

Material examined: 1 male, Montagna Grande (Cosenza), 1235 m, 25.VII.2016, leg. Scalercio and Infusino.

Chorotype: Palaearctic.

Notocelia rosaecolana (Doubleday, 1850)

Material examined: 1 male, Saracena (Cosenza), 1010 m, 20.V.2015, leg. Scalercio and Infusino; 1 male, Aprigliano (Cosenza), 1310 m, 15.VI.2015, leg. Scalercio and Infusino; 
1 male, Saracena (Cosenza), 1010 m, 19.VI.2015, leg. Scalercio and Infusino; 1 male, Vivaio Sbanditi (Cosenza), $1351 \mathrm{~m}, 6$.VII.2015, leg. Scalercio and Infusino.

Chorotype: Asiatic-European.

Notocelia trimaculana (Haworth, [1811])

Material examined: 2 males, Alessandria del Carretto (Cosenza), 1337 m, 21.VI.2016, leg. Scalercio and Infusino. Chorotype: W-Palaearctic.

Blastesthia tessulatana (Staudinger, 1871)

Material examined: 3 males, Rende (Cosenza), 205 m, 14.V.2014, leg. Scalercio.

Chorotype: S-European-Mediterranean.

Rhyacionia buoliana ([Denis and Schiffermüller], 1775) Material examined: 1 female, Aieta (Cosenza), $500 \mathrm{~m}$, 12.VII.1989, leg. Hausmann.

Chorotype: Holarctic.

Rhyacionia pinicolana (Doubleday, 1849)

Material examined: 1 male, Lago Cecita (Cosenza), 1163 m, 30.VII.2013, leg. Scalercio; 2 females, Fossiata (Cosenza), 1300 m, 13.VIII.2013, leg. Scalercio; 1 female, Lago Cecita (Cosenza), 1163 m, 13.VIII.2014, leg. Scalercio; 1 male, Montagna Grande (Cosenza), 1235 m, 25.VII.2016, leg. Scalercio and Infusino.

Chorotype: Asiatic-European.

Rhyacionia pinivorana (Lienig and Zeller, 1846)

Material examined: 1 male, Vivaio Sbanditi (Cosenza), 1354 m, 5.VI.2015, leg. Scalercio and Infusino; 1 male, Aprigliano (Cosenza), 1310 m, 15.VI.2015, leg. Scalercio and Infusino; 1 male, Mangiatoie (Cosenza), $1275 \mathrm{~m}$, 11.V.2016, leg. Scalercio and Infusino; 1 male, Montagna Grande (Cosenza), 1370 m, 11.V.2016, leg. Scalercio and Infusino; 1 male, Mangiatoie (Cosenza), $1275 \mathrm{~m}$, 7.VI.2016, leg. Scalercio and Infusino; 1 male, San Bernardo (Cosenza), 1383 m, 7.VI.2016, leg. Scalercio and Infusino.

Chorotype: Asiatic-European.

Clavigesta sylvestrana (Curtis, 1850)

Material examined: 2 males, Lago Cecita (Cosenza), 1163 m, 13.VIII.2013, leg. Scalercio; 1 male, Fossiata (Cosenza), 1300 m, 13.VIII.2013, leg. Scalercio; 8 males, Lago Cecita (Cosenza), 1176 m, 2.IX.2013, leg. Scalercio; 5 males and 2 females, Lago Cecita (Cosenza), 1163 m, 13.VIII.2014, leg. Scalercio; 1 male, Diga del Menta (Reggio Calabria), 1380 m, 26.VIII.2016, leg. Scalercio.

Chorotype: European.

\section{Tribe Grapholitini}

* Cydia semicinctana (Kennel, 1901)

Records: Sersale (Catanzaro), 1.VI.1985; Sersale (Catanzaro), 31.VIII.1985; Sersale (Catanzaro), 10.V.1986; Sersale (Catanzaro), 16.VIII.1986; Perticaro (Catanzaro), 6.VII-3.VIII.1988; Mendicino (Cosenza), 1-15.VIII.1988 (in TREMATERRA et al., 1994).

Chorotype: Turanic-European.

Cydia succedana ([Denis and Schiffermüller], 1775)

Material examined: 1 male, Righio (Cosenza), $1355 \mathrm{~m}$, 23.V.2014, leg. Scalercio; 1 male, Vivaio Sbanditi (Cosenza), 1350 m, 26.VI.2014, leg. Scalercio; 1 male,
Montagna Grande (Cosenza), 1355 m, 11.V.2015, leg. Scalercio and Infusino; 1 male, Montagna Grande (Cosenza), 1355 m, 18.V.2015, leg. Scalercio and Infusino; 1 male, S.S. Bruno (Vibo Valentia), 940 m, 26.V.2015, leg. Scalercio and Infusino; 2 males, S.S. Bruno (Vibo Valentia), 1040 m, 26.V.2015, leg. Scalercio and Infusino; 1 male, Fiego di San Fili (Cosenza), 740 m, 28.V.2015, leg. Scalercio and Infusino; 1 male, Vivaio Sbanditi (Cosenza), 1351 m, 15.VI.2015, leg. Scalercio and Infusino; 1 male, Pedace (Cosenza), 1440 m, 15.VI.2015, leg. Scalercio and Infusino; 1 male, S.S. Bruno (Vibo Valentia), $830 \mathrm{~m}$, 10.V.2016, leg. Scalercio and Infusino; 1 male, S.S. Bruno (Vibo Valentia), $840 \mathrm{~m}, 10 . \mathrm{V} .2016$, leg. Scalercio and Infusino; 1 male, Mangiatoie (Cosenza), $1270 \mathrm{~m}$, 11.V.2016, leg. Scalercio and Infusino; 1 male, S.S. Bruno (Vibo Valentia), $840 \mathrm{~m}, 30 . \mathrm{V} .2016$, leg. Scalercio and Infusino; 1 male, S.S. Bruno (Vibo Valentia), $925 \mathrm{~m}$, 30.V.2016, leg. Scalercio and Infusino; 4 males, San Fili (Cosenza), 831 m, 25.V.2017, leg. Colacci, Goglia and Scalercio; 6 males and 1 female, San Nicola Silano (Cosenza), 1435 m, 17.V.2018, leg. Colacci and Goglia; 1 female, Rovale (Cosenza), 1440 m, 17.V.2018, leg. Colacci and Goglia.

Chorotype: Centro Asiatic-European-Mediterranean.

Cydia ulicetana (Haworth, [1811])

Material examined: 1 male, San Fili (Cosenza), 831 m, 25.V.2017, leg. Colacci, Goglia and Scalercio.

Chorotype: European-Mediterranean.

Cydia trogodana Pröse, 1988

Material examined: 1 male, Palizzi Marina (Reggio Calabria), 3 m, 15.V.2015, leg. Urso.

Chorotype: E-Mediterranean.

* Cydia coniferana (Saxesen, 1840)

Records: Sersale (Catanzaro), 15.VI-31.VIII.1985; Sersale (Catanzaro), 15.VI-31.VIII.1986 (in TREMATERRA et al., 1994).

Chorotype: Sibiric-European.

Cydia pomonella (Linnaeus, 1758)

Material examined: 1 male, Pianette di Dipignano (Cosenza), 765 m, 29.VII.2013, leg. Scalercio; 2 males and 1 female, Pianette di Dipignano (Cosenza), $765 \mathrm{~m}$, 5.VIII.2013, leg. Scalercio; 1 male, Montalto Uffugo (Cosenza), 230 m, 24.V.2014, leg. Scalercio.

Chorotype: W-Palaearctic.

Cydia triangulella (Goeze, 1783)

Material examined: 1 female, S.B. Ullano (Cosenza), 893 m, 29.VIII.2016, leg. Scalercio and Infusino.

Chorotype: Palaearctic.

Cydia fagiglandana (Zeller, 1841)

Material examined: 1 male, Pianette di Dipignano (Cosenza), 765 m, 5.VIII.2013, leg. Scalercio; 1 male, Donnici (Cosenza), 550 m, 7.VIII.2013, leg. Scalercio; 1 female, Lago Cecita (Cosenza), 1163 m, 13.VIII.2013, leg. Scalercio; 1 female, Vivaio Sbanditi (Cosenza), $1350 \mathrm{~m}$, 26.VI.2014, leg. Scalercio; 1 male, Lago Cecita (Cosenza), 1163 m, 13.VIII.2014, leg. Scalercio; 1 female, Saracena (Cosenza), 1010 m, 20.V.2015, leg. Scalercio and Infusino; 1 male, Saracena (Cosenza), 1010 m, 19.VI.2015, leg. Scalercio and Infusino; 1 male, Saracena (Cosenza), 1460 m, 20.VII.2015, leg. Scalercio and Infusino; 2 females, Montagna Grande (Cosenza), 1355 m, 25.VII.2016, leg. 
Scalercio and Infusino; 1 female, Lungro (Cosenza), 1270 m, 1.VIII.2016, leg. Scalercio and Infusino; 1 female, Acquaformosa (Cosenza), 1357 m, 1.VIII.2016, leg. Scalercio and Infusino; 1 female, Montalto Uffugo (Cosenza), 545 m, 29.VIII.2016, leg. Scalercio and Infusino; 1 female, S.B. Ullano (Cosenza), $731 \mathrm{~m}$, 29.VIII.2016, leg. Scalercio and Infusino.

Chorotype: W-Palaearctic.

\section{Cydia amplana (Hübner, [1796-1799])}

Material examined: 1 female, f. Argentino (Cosenza), 250 m, 4.IX.1991, leg. Hausmann.

Chorotype: Turanic-European.

Lathronympha strigana (Fabricius, 1775)

Material examined: 1 male, Aprigliano (Cosenza), 1310 m, 15.VI.2015, leg. Scalercio and Infusino; 1 male, Alessandria del Carretto (Cosenza), 1291 m, 21.VI.2017, leg. Scalercio and Infusino.

Chorotype: Sibiric-European.

Selania leplastriana (Curtis, 1831)

Material examined: 1 female, Sangineto (Cosenza), $50 \mathrm{~m}$, 10.VIII.2014, leg. Scalercio.

Chorotype: European-Mediterranean.

Selania capparidana (Zeller, 1847)

Material examined: 4 males and 4 females, Copanello (Catanzaro), 70 m, 25.VII.1996, leg. ?; 4 males and 4 females, Copanello (Catanzaro), 70 m, 10.VIII.1996, leg. Trematerra.

Chorotype: Mediterranean.

Grapholita fissana (Frölich, 1828)

Material examined: 1 male, S.B. Ullano (Cosenza), 893 m, 1.VI.2016, leg. Scalercio and Infusino.

Chorotype: European.

Grapholita difficilana (Walsingam, 1900)

Material examined: 1 male, Cozzi dell'Anticristo (Cosenza), 1300 m, 28.V.1990, leg. Trematerra; 1 female, lago Ampollino (Cosenza), 1300 m, 11.VI.1991, leg. Trematerra; 5 females, Cozzi dell'Anticristo (Cosenza), 1300 m, 13.VI.1991, leg. Trematerra.

Chorotype: European-Mediterranean.

Grapholita lunulana ([Denis and Schiffermüller], 1775)

Material examined: 1 female, Donnici (Cosenza), 550 m, 18.V.2014, leg. Scalercio.

Chorotype: Centro Asiatic-European-Mediterranean.

* Grapholita orobana Treitschke, 1830

Records: Sersale (Catanzaro), 15.VI-6.VII.1985; Sersale (Catanzaro), 15.VI-6.VII.1986; Soveria Mannelli (Catanzaro), 4.VI-23.VII.1987; Soveria Mannelli (Catanzaro), 4.VI-23.VII.1988; Mendicino (Cosenza), 620.VII.1987; Mendicino (Cosenza), 27.VI-4.VII.1988; Monte Cerviero (Cosenza), 28.V.1989 (in TREMATERRA et al., 1994).

Chorotype: Sibiric-European.

* Grapholita gemmiferana Treitschke, 1835

Records: Sersale (Catanzaro), 28.V-22.VI.1985; Sersale (Catanzaro), 10.V.1986; Sersale (Catanzaro), 6.VII.1986; Soveria Mannelli (Catanzaro), 4-25.VI.1987 (in TREMATERRA et al., 1994).

Chorotype: Centro Asiatic-European.
Grapholita lathyrana (Hübner, [1811-1813])

Material examined: 1 male, Gariglione (Catanzaro), 1600 m, 12.VI.1991, leg. Trematerra.

Chorotype: European.

* Grapholita funebrana Treitschke, 1835

Records: Sersale (Catanzaro), 22.VI-14.IX.1985; Sersale (Catanzaro), 17.V-16.VIII.1986; Soveria Mannelli (Catanzaro), 4.VI-10.IX.1987; Mendicino (Cosenza), 22.VI-21.IX.1987; Soveria Mannelli (Catanzaro), 4.VI10.IX.1988; Mendicino (Cosenza), 22.VI-21.IX.1988; Perticaro (Catanzaro), 24.VI-20.VIII.1988; Mendicino (Cosenza), 22.VI-21.IX.1989 (in TREMATERRA et al., 1994).

Chorotype: Palaearctic.

* Grapholita janthinana (Duponchel, 1835)

Records: Sersale (Catanzaro), 12.VII-2.VIII.1986; Mendicino (Cosenza), 20.VII.1987 (in TREMATERRA et al., 1994).

Chorotype: Turanic-European

* Grapholita tenebrosana Duponchel, 1843

Records: Sersale (Catanzaro), 15.VI-27.VII.1985; Sersale (Catanzaro), 15.VI-27.VII.1986; Soveria Mannelli (Catanzaro), 4.VI-2.VII.1987; Soveria Mannelli (Catanzaro), 4.VI-2.VII.1988; Mendicino (Cosenza), 29.VI.1989 (in TREMATERRA et al., 1994).

Chorotype: Asiatic-European.

Pammene amygdalana (Duponchel, 1842)

Material examined: 1 male, Pianette di Dipignano (Cosenza), 765 m, 29.VII.2013, leg. Scalercio.

Chorotype: European-Mediterranean.

\section{Pammene querceti Gozmány, 1957}

Material: Sersale (Catanzaro), 25.V.1985; Sersale (Catanzaro), 10.VIII.1985; Sersale (Catanzaro), 10.V-7.VI.1986 (in TREMATERRA et al., 1994).

Chorotype: S-European.

\section{Pammene fasciana (Linnaeus, 1761)}

Material examined: 1 male, Fiego di San Fili (Cosenza), 740 m, 25.VI.2015, leg. Scalercio and Infusino; 2 females, S.B. Ullano (Cosenza), 825 m, 27.VII.2016, leg. Scalercio and Infusino; 2 males, Montalto Uffugo (Cosenza), $550 \mathrm{~m}$, 29.VIII.2016, leg. Scalercio and Infusino.

Chorotype: Turanic-European.

* Pammene argyrana (Hübner, 1799)

Records: Sersale (Catanzaro) 13.VII-17.VIII.1985 (in TREMATERRA et al., 1994).

Chorotype: European

Remarks: collected in July-August, previously reported in TREMATERRA (2003) from April to June.

* Pammene albuginana (Guenée, 1845)

Records: Sersale (Catanzaro), 14.IX.1985; Sersale (Catanzaro), 17.V.1986; Soveria Mannelli (Catanzaro), 4.VI.1987; Mendicino (Cosenza), 28.IX-19.X.1987; Soveria Mannelli (Catanzaro), 1.X.1987 (in TREMATERRA et al., 1994).

Chorotype: European.

* Pammene gallicolana (Lienig and Zeller, 1846)

Records: Sersale (Catanzaro), 29.VI-5.X.1985; Sersale (Catanzaro), 10.V-2.VIII.1986; Mendicino (Cosenza), 1.VI12.X.1987; Soveria Mannelli (Catanzaro), 4.VI-8.X.1987; 
Mendicino (Cosenza), 1.VI-12.X.1988; Perticaro (Catanzaro), 20.VII.1988; Mendicino (Cosenza), 1.VI12.X.1989 (in TREMATERRA et al., 1994).

Chorotype: European.

* Pammene spiniana (Duponchel, 1843)

Records: Soveria Mannelli (Catanzaro), 20.VIII8.X.1987; Mendicino (Cosenza), 21.IX-19.X.1987 (in TREMATERRA et al., 1994).

Chorotype: European-Mediterranean.

* Pammene blockiana (Herrich-Schäffer, 1851)

Records: Sersale (Catanzaro), 19.VII-2.VIII.1986 (in TREMATERRA et al., 1994).

Chorotype: S-European.

* Pammene aurana (Fabricius, 1775)

Records: Sersale (Catanzaro), 20.VII.1985; Soveria Mannelli (Catanzaro), 17.IX.1987 (in TREMATERRA et al., 1994).

Chorotype: Sibiric-European.

Strophedra nitidana (Fabricius, 1794)

Material examined: 1 male, S.S. Bruno (Vibo Valentia), 830 m, 30.V.2016, leg. Scalercio and Infusino.

Chorotype: Asiatic-European.

* Dichrorampha acuminatana (Lienig and Zeller, 1846)

Records: Sersale (Catanzaro), 29.VI.1985; Copanello (Catanzaro), 24.IV.1992, leg. Trematerra (in TREMATERRA et al., 1994).

Chorotype: European (extension Anatolian-Maghrebian).

Dichrorampha letarfensis Gibeaux, 1983

Material examined: 4 males and 5 females, Copanello (Catanzaro), 20 m, 24.IV.1992, leg. Trematerra.

Chorotype: W-Mediterranean.

Dichrorampha gemellana (Zeller, 1847)

Material examined: 1 male, Lago Cecita (Cosenza), 1176 m, 2.IX.2013, leg. Scalercio.

Chorotype: Tirrenic.

\section{CONSIDERATIONS ON THE TORTRICIDAE FAUNA OF CALABRIA}

During our entomological expeditions realized from 2013 to 2018 in Calabria region, 123 species of Lepidoptera Tortricidae were caught, to which we added 22 species deposited in the Trematerra Collection and other 36 species reported by Trematerra in the course of entomological researches carried out in the past in the Calabrian territory, up to a total of 181 taxa of the family.

Species belonging to all the tribes cited in the Italiana fauna (TREMATERRA, 2003) have been found in Calabria, with the exception of the members of the Sparganothini tribe (subfamily Tortricinae), the two most numerous tortricids subfamilies recorded are the Tortricinae and the Olethreutinae, which has 75 species and 102 species, respectively; the subfamily Chlidanotinae is represented by 4 taxa.

A biogeographic study on the Tortricidae found in Calabria, using chorological analysis, are reported in Table 2. It can be noted that there are few Cosmopolitan species (1.10\%), Subcosmopolitan (2.21\%) and Holartic (9.94\%). More than $59 \%$ of the species show a wide Palaearctic
Table 2 - Chorological categories, number of species and percentages of Lepidoptera Tortricidae collected in Calabria (southern Italy).

\begin{tabular}{|c|c|c|}
\hline CHOROTYPE & $\begin{array}{l}\text { N. OF } \\
\text { SPECIES }\end{array}$ & $\%$ \\
\hline $\begin{array}{l}\text { Cosmopolitan and Subcosmopolitan } \\
\text { distribution }\end{array}$ & 6 & 3.31 \\
\hline Cosmopolitan & 2 & 1.10 \\
\hline Subcosmopolitan & 4 & 2.21 \\
\hline Holarctic distribution & 18 & 9.94 \\
\hline Holarctic & 18 & 9.94 \\
\hline Wide Palaearctic distribution & 107 & 59.12 \\
\hline $\begin{array}{r}\text { Palaearctic } \\
\end{array}$ & 12 & 6.63 \\
\hline W-Palaearctic & 14 & 7.73 \\
\hline Asiatic-European & 32 & 17.68 \\
\hline Sibiric-European & 8 & 4.42 \\
\hline $\begin{array}{r}\text { Centro Asiatic-European-Mediterra- } \\
\text { nean }\end{array}$ & 8 & 4.42 \\
\hline Centro Asiatic-European & 10 & 5.52 \\
\hline Turanic-European-Mediterranean & 4 & 2.22 \\
\hline Turanic-European & 12 & 6.63 \\
\hline European-Mediterranean & 6 & 3.31 \\
\hline $\begin{array}{r}\text { European (extension Anatolian- } \\
\text { Maghrebian) }\end{array}$ & 1 & 0.55 \\
\hline European distribution & 34 & 18.78 \\
\hline European & 24 & 13.26 \\
\hline Central European & 1 & 0.55 \\
\hline S-European & 9 & 4.97 \\
\hline Mediterranean distribution & 12 & 6.63 \\
\hline Mediterranean & 4 & 2.21 \\
\hline S-European-Mediterranean & 1 & 0.55 \\
\hline W-Mediterranean & 3 & 1.66 \\
\hline E-Mediterranean & 1 & 0.55 \\
\hline Tirrenic & 1 & 0.55 \\
\hline Apenninic-Dinaric & 1 & 0.55 \\
\hline $\begin{array}{r}\text { NW-African (extension S-Apenninic- } \\
\text { Sicilian) }\end{array}$ & 1 & 0.55 \\
\hline Endemic Italian species & 4 & 2.21 \\
\hline Apenninic & 2 & 1.10 \\
\hline S-Apenninic & 2 & 1.10 \\
\hline
\end{tabular}

distribution. In this grouping, the species with the chorotypes Asiatic-European (17.68\%), W-Palaearctic $(7.73 \%)$, Palaearctic $(6.63 \%)$ and Turanic-European $(6.63 \%)$ are well represented. Fewer are the Centro AsiaticEuropean (5.52\%), Centro Asiatic-European-Mediterranean $(4.42 \%)$, Sibiric-European (4.42\%), EuropeanMediterranean (3.31\%), Turanic-European-Mediterranean $(2.22 \%)$ and European (with extension AnatolianMaghrebian) $(0.55 \%)$.

More than $18 \%$ of the collected taxa are tortricids with European distribution; the elements belonging to this chorotype show European (13.26\%), S-European (4.97\%) and Central European $(0.55 \%)$ distribution. The Mediterranean distribution taxa is shown only by $6.63 \%$ of the recorded species including Mediterranean (2.21\%), W- 
Mediterranean (1.66\%) and, all with $0.55 \%$, S-EuropeanMediterranean, E-Mediterranean, Tirrenic, Apenninic-Dinaric, NW-African (with extension S-ApenninicSicilian) elements. Finally, the presence of two species with Apennine chorotype and two with S-Apennines chorotype is reported.

\section{COMMENTS}

The fauna of Lepidoptera Tortricidae in the Calabrian study areas is dominated by forest elements, on the contrary, only few are agrarian elements. Many species are related to arboreal and uncultivated plants; the latter have been expanding on lands due to the abandonment of the fields and the continuous deforestation. The increase in plant cover favors the increase of lepidopteran populations (of all families) with mesophilic or nemoral behaviour.

Unlike what has occurred for other Italian regions, the tortricids present in the southern areas of the country have received little or sporadic attention from Italian and foreign entomologists. Overall information on this matter can be found in the Checklist of the Italian fauna (TREMATERRA, 1995) and in the "Catalogue of Lepidoptera Tortricidae of the Italian fauna" (TREMATERRA, 2003).

In the present report, 59 of the whole species (over $30 \%$ of the total) were found for the first time in the Calabria: Aleimma loeflingianum, Acleris abietana, Acleris sparsana, Acleris rhombana, Acleris schalleriana, Acleris cristana, Acleris aspersana, Acleris hastiana, Acleris notana, Acleris ferrugana, Acleris quercinana, Acleris literana, Phtheochroa rugosana, Phalonidia gilvicomana, Phalonidia manniana, Agapeta hamana, Eupoecilia angustana, Aethes cnicana, Cochylis salebrana, Cochylis molliculana, Eana penziana, Archicnephasia hartigi, Cnephasia fulturata, Cnephasia hellenica, Batodes angustiorana, Paramesia diffusana, Archips crataeganus, Choristoneura diversana, Argyrotaenia ljungiana, Ptycoloma lecheana, Aphelia ferugana, Isotrias rectifasciana, Bactra lancealana, Bactra venosana, Endothenia nigricostana, Endothenia pullana, Piniphila bifasciana, Syricoris rivulana, Ancylis comptana, Ancylis paludana, Ancylis badiana, Ancylis mitterbacheriana, Rhopobota naevana, Epinotia sordidana, Epinotia solandriana, Epinotia fraternana, Pelochrista subtiliana, Eucosma hohenwartiana, Eucosma balatonana, Eucosma albidulana, Epiblema graphana, Notocelia aquana, Notocelia rosaecolana, Notocelia trimaculana, Rhyacionia pinivorana, Cydia trogodana, Grapholita fissana, Pammene amygdalana, and Strophedra nitidana.

Our findings furthermore revealed 23 species that have as a southern limit of their distribution area the Calabrian territory: Acleris notana, Phtheochroa purana, Eana italica, Archicnephasia hartigi, Cnephasia zangheriana, Choristoneura murinana, Dichelia histrionana, Olindia schumacherana, Endothenia lapideana, Endothenia pullana, Syricoris lacunana, Ancylis paludana, Spilonota laricana, Epinotia signatana, Epinotia nigricana, Pelochrista subtiliana, Eucosma balatonana, Gypsonoma sociana, Rhyacionia pinivorana, Cydia coniferana, Pammene querceti, Pammene albuginana, and Pammene aurana.

Finally, the finding of the two Apennine endemisms, Cnephasia zangheriana and Isotrias joannisana, and two SApennines species, Isotrias martelliana and Archicnephasia hartigi, is of particular interest.

Overall it is possible to observe that a limited number of specimens belonging to entities potentially harmful to agricultural and forest plants was recorded in Calabria, especially in the northern and central part of the territory, this suggests that these areas may in good health and maintains a high degree of naturalness. However, the absence of tortricid pests could be also due to the particular natural habitats visited during the entomological expeditions.

\section{ACKNOWLEDGEMENTS}

We are grateful to dr Marco Infusino and dr Silvia Greco (Consiglio per la Ricerca in agricoltura e l'analisi dell'Economia Agraria, Rende, Cosenza, Italy) for their help in fieldwork. The investigation was funded as part of the "Monitoring Project for Nocturnal Lepidoptera through the use of Rothamsted light traps", by the Sila National Park at the Research Center for Forestry and Wood in Rende, and of the Project "ALForLab" (PON03PE_00024_1) cofunded by the National Operational Programme for Research and Competitiveness (PON R\&C) 2007-2013, through the European Regional Development Fund and National Resource [Revolving Fund - Cohesion Action Plan, MIUR].

\section{REFERENCES}

AARVIK L.E., 2013 - Tortricidae. - In: Karsholt O. \& Van Nieukerken E.J. Fauna Europaea version 2.6.2. Available from http://www.faunaeur.org.

Brown J.W., 2005 - World Catalogue of Insects. Volume 5. Tortricidae (Lepidoptera). - Apollo Books. Stenstrup: 1741.

Gilligan T.M., BaiXeras J., Brown J.W., TucK K.R., 2014 - T@RTS: Online World Catalogue of the Tortricidae (Ver. 3.0). http://www.tortricid.net/catalogue.asp

Infusino M., Brehm G., Di Marco C., SCAlercio S., 2017 - Assessing the efficiency of UV LEDs as light sources for sampling the diversity of macro-moth. - European Journal of Entomology, 114: 25-33.

Parenzan P., 1994 - Proposta di codificazione per una gestione informatica dei corotipi $W$-Paleartici, con particolare riferimento alla fauna italiana. Entomologica, 28: 93-98.

Razowski J., 1970 - Cochylidae. In: Amsel H.G., Gregor F., Reisser H., Microlepidoptera Palearctica. - Verlag Georg Fromme \& Co., Wien: 1-528.

RAZOWSKI J., 2002 - Tortricidae of Europe. Volume 1. Tortricinae and Chlidanotinae. - František Slamka. Bratislava: 1-247.

RAZOWSKI J., 2003 - Tortricidae of Europe. Volume 2. Olethreutinae. - František Slamka. Bratislava: 1-301.

TrematerRA P., 1990 - Isotrias martelliana sp. $\mathrm{n}$. (Lepidoptera Tortricidae) rinvenuta sul Massiccio del Pollino. - Bollettino di Zoologia agraria e di Bachicoltura Ser. II, 22 (1): 1-5.

Trematerra P., 1991a - Lepidotteri Tortricidi nel Massiccio del Pollino. - Atti XVI Congresso nazionale italiano di entomologia, Bari-Martina Franca (TA): 207 214

Trematerra P., 1991b - I Cochylini della collezione A. Fiori nel Museo di Storia Naturale di Milano. - Memorie della Società Entomologica Italiana, 70 (2): 267-285.

TrematerRA P., 1995 - Lepidoptera Tortricoidea. In: Minelli A., Ruffo S., La Posta S. Checklist delle specie della fauna italiana. - Edizioni Calderini, Bologna: 2-17.

Trematerra P., 2003 - Catalogo dei Lepidoptera 
Tortricidae della fauna italiana: geonemia, distribuzione in Italia, note biologiche, identificazione. - Bollettino di Zoologia agraria e di Bachicoltura, Ser. II, 35 (suppl. 1): $1-270$.

Trematerra P., Colacci M., 2016 - I Tortricidae della Collezione Usvelli (Lepidoptera). - Gortania - Botanica, Zoologia, 37 (2015): 47-81.

Trematerra P., Rotundo G., Giacometti R., 1994 Contributo alla conoscenza dei Tortricidi della Calabria. - Bollettino del Laboratorio di Entomologia agraria Filippo Silvestri, 49 (1992): 3-15.
Trematerra P., Sciarretta A., Parenzan P., 1997 - Note faunistiche su alcuni interessanti Tortricidi dell'Italia meridionale (Lepidoptera). - Entomologica, 31: 181-189.

Vigna Taglianti, A., Audisio P.A., Belfiore C., Biondi M.A., Bologna M.A., Carpaneto G.M., De Biase A., De Felici S., Piattella E., Racheli T., Zapparoli M., Zoia S., 1992 - Riflessioni di gruppo sui corotipi fondamentali della fauna $W$-paleartica ed in particolare italiana. - Biogeographia, 16: 159-210. 
182 - Blank Page 